Spin Physics (SPIN2014)

International Journal of Modern Physics: Conference Series

Vol. 40 (2016) 1660035 (6 pages)

(C) The Author(s)

DOI: 10.1142/S2010194516600351

\title{
Key Future Measurements of TMDs at Jefferson Lab and Other Facilities
}

\author{
Kalyan Allada \\ Laboratory of Nuclear Science, \\ Massachusetts Institute of Technology, \\ 77 Massachusetts Avenue \\ Cambridge, Massachusetts 02139,USA \\ allada@mit.edu
}

Published 29 February 2016

\begin{abstract}
Transverse-momentum dependent parton distribution functions (TMDs) provide a description of nucleon structure in terms of the parton transverse momentum and its transverse spin. At leading twist there are eight TMDs, each offering a unique feature of quarks in a polarized or an unpolarized nucleon. The Sivers distribution is one of the most interesting TMD due to its non-universality. It has been extracted using the data from semi-inclusive deep-inelastic scattering (SIDIS), but there is no data yet from spin-dependent Drell-Yan (DY) process. Such measurement will provide a crucial test of TMD formalism which predicts an equal magnitude and opposite sign for the Sivers function extracted from SIDIS and DY process. We will discuss key future measurements of TMDs using both SIDIS and DY process with a focus on Hall A SoLID SIDIS program at Jefferson Lab.
\end{abstract}

Keywords: TMDs; SIDIS; Drell-Yan.

\section{Introduction}

One of the major goals in the investigation of the nucleon spin structure is the extraction of transverse momentum dependent parton distribution functions (TMDs) using the data obtained from lepton-lepton and hadron-hadron scattering. TMDs provide new and fundamental information about the structure of the nucleon by imaging its partonic structure, dynamics, and spin-orbital couplings in three-momentum space. In the leading twist there are eight TMD quark distributions: ${ }^{1}$ the first three, the unpolarized $\left(f_{1}\right)$, the helicity $\left(g_{1}\right)$ and the transversity $\left(h_{1}\right)$ distributions are integrated over the transverse momenta of quarks, while the other five have an explicit dependence on the transverse momentum of quarks $\left(k_{T}\right)$.

This is an Open Access article published by World Scientific Publishing Company. It is distributed under the terms of the Creative Commons Attribution 3.0 (CC-BY) License. Further distribution of this work is permitted, provided the original work is properly cited. 
The unpolarized and helicity distributions can be accessed in inclusive DIS process and the rest of the TMDs can be probed using semi-inclusive deep inelastic scattering (SIDIS). TMDs can also be probed in hadronic collisions. For example, the azimuthal distribution of charged pions within a jet is sensitive to the convolution of $h_{1}$ with Collins fragmentation function. ${ }^{2}$ Similarly, the azimuthal asymmetries of lepton pair in spin-dependent Drell-Yan (DY) process are sensitive to certain TMDs such as the Sivers and the Boer-Mulders functions. ${ }^{3}$

The transversity distribution gives the probability of finding a transversely polarized parton inside a transversely polarized nucleon with a longitudinal momentum fraction $x$ and transverse momentum $k_{T}$. The first moment of $h_{1}$ integrated over the full range of $x$ gives the nucleon tensor charge, which is an important quantity in QCD and calculable on the lattice. The Sivers function $\left(f_{1 T}^{\perp}\right)^{4}$ provides the number density of unpolarized partons inside a transversely polarized proton, and it requires wave function components with nonzero orbital angular momentum and thus provides information about the correlation between the quark orbital angular momentum (OAM) and nucleon spin. Furthermore, it is a (naive) T-odd function which relies on the final state interactions (FSI) experienced by the active quark in a SIDIS process. It was shown using the parity and time reversal invariance in QCD that the quark Sivers function in SIDIS and that in the DY process are equal in magnitude and opposite in sign to each other. ${ }^{5-7}$ This sign change of the Sivers functions between SIDIS and DY is one of the important predictions and a critical test of the QCD factorization formalism. In contrast to $f_{1 T}^{\perp}$, the worm-gear functions $g_{1 T}$ and $h_{1 L}^{\perp}$ are T-even, and thus do not require FSI to be nonzero. Nevertheless, they also require interference between wave function components that differ by one unit of OAM and thus require OAM to be nonzero. Finally, pretzelosity $\left(h_{1 T}^{\perp}\right)$ requires interference between wave function components that differ by two units of OAM (e.g. p-p or s-d interference). The combined information from all these functions could thus be invaluable for disentangling the spin orbit correlations in the nucleon wave function, providing important information about the quark orbital angular momentum, and for imaging of the nucleon in full momentum space.

Single spin asymmetries (SSAs) in SIDIS using a transversely polarized proton target were measured by both $\operatorname{HERMES}^{8,9}$ and $\mathrm{COMPASS}^{10}$ collaborations. Non-zero asymmetries were observed for both Collins and Sivers moments in the $\pi^{+}$channel. However, for the $\pi^{-}$channel the Collins asymmetry was non-zero, whereas Sivers asymmetry was small and consistent with zero within the statistical precision of these experiments. Jefferson Lab E06-010 experiment measured SSAs on a polarized ${ }^{3} \mathrm{He}$ target to extract Collins and Sivers moments for charged pions $^{11}$ and kaons, ${ }^{12}$ and double spin asymmetries (DSA) needed to extract the $g_{1 T}$ TMD. ${ }^{13}$ More recently, the pretzelosity asymmetry ${ }^{14}$ and SSAs in inclusive hadron production ${ }^{15}$ were also extracted from this experiment. The planned future measurements at Jefferson Lab will significantly increase the precision of these measurement, extending it to a much wider kinematic range. 


\section{Future TMD Measurements at Jefferson Lab}

Jefferson Lab has a comprehensive SIDIS program in three Halls (A, B and C) using the upgraded $12 \mathrm{GeV}$ accelerator. While Hall A and B plans to measure SSAs and DSAs using polarized targets, Hall $\mathrm{C}$ will focus on precision measurement of absolute SIDIS cross-sections using unpolarized hydrogen and deuterium targets. Hall A SIDIS program consists of two distinct devices - one using the combination of the BitBite and the new Super BiteBite Spectrometer (SBS), and the other using the proposed Solenodial Large Intensity Device (SoLID). There is currently one SIDIS proposal using the SBS which plans to measure the spin asymmetries of charged pions and kaons using transversely polarized ${ }^{3} \mathrm{He}$ target. ${ }^{16}$ The SIDIS program with SoLID spectrometer involves several proposals to measure the TMD related spin asymmetries using polarized ${ }^{3} \mathrm{He}$ and proton targets. A detailed discussion of the SoLID program is provided in the section 2.1. Hall B will use CLAS12 spectrometer with both polarized and unpolarized targets to measure spin asymmetries for charged pions and kaons. The CLAS12 SIDIS program is very comprehensive and consists of several proposals. In particular, there are plans to measure the Collins and Sivers moments using transversely polarized proton target, ${ }^{17}$ and SSAs in di-hadron production which are sensitive to the transversity distribution. ${ }^{18}$ The Hall C SIDIS experiments will use the High Momentum Spectrometer (HMS) and the Super-HMS (SHMS). The main focus will be to measure the $\pi^{+} / \pi^{-}$ratios, ${ }^{19}$ $\mathrm{R}_{S I D I S}=\sigma_{L} / \sigma_{T}{ }^{20}$ and hadron transverse momentum $\left(p_{T}\right)$ dependence in the SIDIS process. ${ }^{21}$

\subsection{Hall A SoLID-SIDIS Setup}

The proposed experimental setup consists of the Solenoidal Large Intensity Device (SoLID) and a polarized target as shown in Fig. 1. The SoLID spectrometer has a full $2 \pi$ azimuthal angular coverage, which is essential to control the systematic

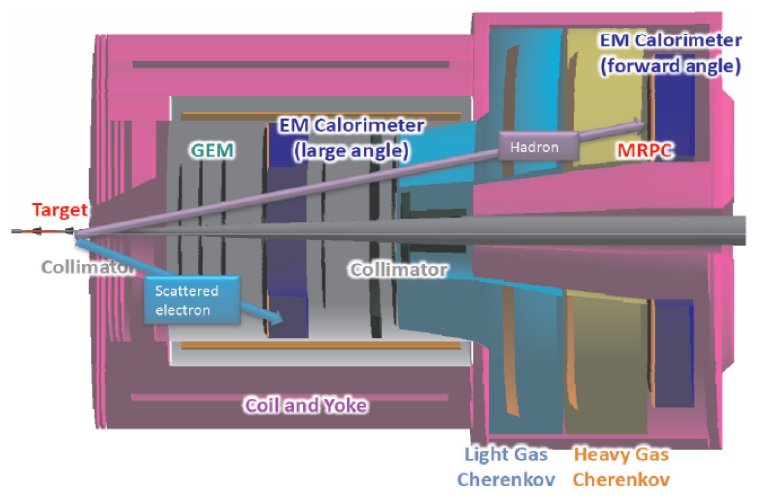

Fig. 1. The experimental layout of the SoLID and polarized target in the proposed measurement. The subdetectors are labeled with colors corresponding to the detector regions. 
uncertainties in extracting various azimuthal moments from the measured asymmetries. The SIDIS program consist of SSAs/DSAs measurements for charged pions using polarized ${ }^{3} \mathrm{He}$ (neutron) ${ }^{22,23}$ and $\mathrm{NH}_{3}$ (proton) targets. ${ }^{24}$ These experiments will also allow for the measurement of SSAs in di-hadron production in SIDIS which are sensitive to the transversity distribution. The polarized luminosity for the ${ }^{3} \mathrm{He}$ target is about $10^{36} \mathrm{~N} \mathrm{~cm}^{-2} \mathrm{~s}^{-1}$ while that for $\mathrm{NH}_{3}$ target it is $10^{35} \mathrm{~N} \mathrm{~cm}^{-2} \mathrm{~s}^{-1}$. Two different beam energies, $11 \mathrm{GeV}$ and $8.8 \mathrm{GeV}$, will be used to measure the asymmetries. The lower beam energy will provide precision data on the radiative corrections along with increased $\mathrm{Q}^{2}$ coverage.

The SoLID detector consists of two regions. The forward region with polar angle coverage of $8^{\circ}$ to $14.8^{\circ}$ has a total solid angle of about $90 \mathrm{msr}$, and covers a momentum range from $0.8 \mathrm{GeV} / \mathrm{c}$ to $7 \mathrm{GeV} / \mathrm{c}$ for both electrons and pions. Tracking will be done by GEM detectors and particle identification will be provided by a combination of a heavy gas and light gas Cerenkov detectors, along with a Shashlyk-type electromagnetic calorimeter. A thin layer of scintillator pad detector (SPD) and one layer of Multi-gap Resistive Plate Chamber (MRPC) will be used for the timing information. The large angle region with polar angle coverage of $16^{\circ}$ to $24^{\circ}$ has a solid angle of about $260 \mathrm{msr}$, and is mainly used for the electron detection in the momentum range of 3.5 to $6 \mathrm{GeV} / \mathrm{c}$. GEMs and an electromagnetic calorimeter will be used in this region to provide accurate momentum and energy reconstruction of the scattered electron where the $\pi^{-} / e$ ratio is expected to be lower than 1.5 .

Figure 2 shows the projected results for the $\pi^{+}$Collins asymmetry in one bin of $Q^{2}$ and $z$ that can be obtained in 90 days with a ${ }^{3} \mathrm{He}$ target. The theoretical predictions of Collins asymmetries are from Anselmino et al. ${ }^{25}$ Vogelsang and Yuan ${ }^{26}$

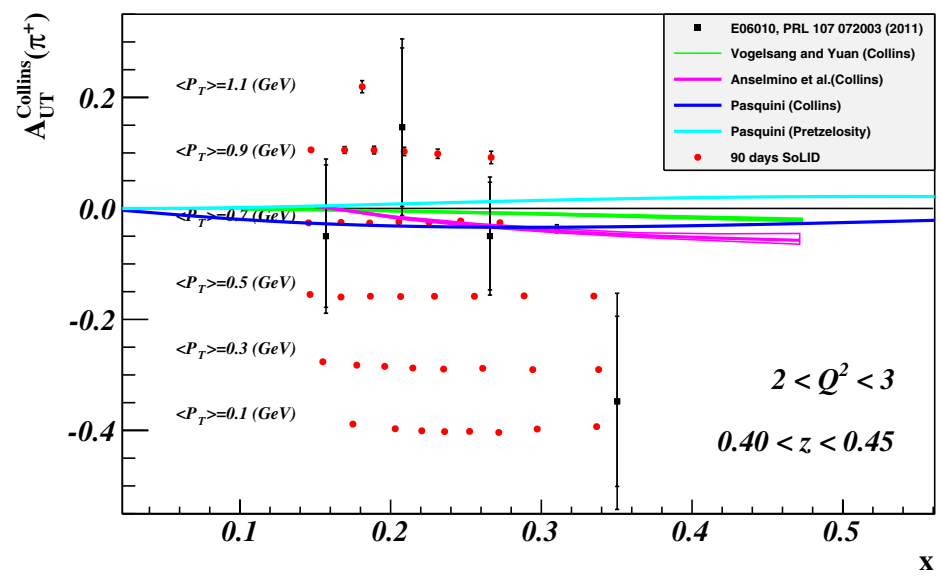

Fig. 2. Projected statistical precision for $\pi^{+}$Collins asymmetry in one bin of $Q^{2}$ and $z$. The $P_{T}$ coverage is shown in different bands of data points and the y-axis shows the scale of the asymmetry. The data from the Hall-A $6 \mathrm{GeV}$ experiment (E06-010) are shown in black with consisting of large errors. 
and Pasquini. ${ }^{27}$ The $\mathrm{x}$-axis shows the bjorken- $x$ and the $\mathrm{y}$-axis shows the scale of the asymmetries. The overall kinematic coverage of SoLID is as follows: $P_{T}$ from 0-1.2 $\mathrm{GeV} / \mathrm{c}, Q^{2}$ from $1-8(\mathrm{GeV} / \mathrm{c})^{2}, z$ from $0.3-0.7$, and $x$ from 0.05-0.6. $P_{T}$ and $z$ coverage is divided into 8 bins each, and the $Q^{2}$ range is divided into 6 bins. The combined data from two beam energies will be divided into a total of 1400 bins for the SIDIS measurement with a ${ }^{3} \mathrm{He}$ target. This will allow us to study the measured asymmetries in 4 dimension $\left(x, Q^{2}, z, P_{T}\right)$. Along with the SSA, the DSA data will allow us to measure $A_{L T}$, which is related to the $g_{1 T}$ distribution. Moreover, SIDIS with longitudinally polarized beam on a longitudinally polarized target will provide $A_{L L}$ and $A_{U L}$ data which will be used to extract $g_{1 L}$ and $h_{1 L}^{\perp}$, respectively.

\section{Future TMD Measurements in Hadronic Collisions}

Currently there are several proposed experiments to measure TMDs in hadronic collisions. Here we discuss a few proposals which focuses on the measurement of Sivers asymmetry to verify the predicted sign change between Drell-Yan and SIDIS process. The COMPASS Drell-Yan experiment will use $190 \mathrm{GeV} \pi^{-}$beam on a transversely polarized proton target. ${ }^{28}$ This experiment will cover valence quark region and plans to measure both the magnitude and the sign of the Sivers and Boer-Mulders asymmetry. There are two proposed experiments at Fermilab that plans to use the $120 \mathrm{GeV}$ proton beam from the main injector. The first one (E1039) plans to use the $120 \mathrm{GeV}$ proton beam on a transversely polarized proton target to measure the sign and magnitude of sea-quark Sivers asymmetry, ${ }^{29}$ and the second experiment (E-1027) plans to polarized the $120 \mathrm{GeV}$ proton beam and use it with an unpolarized proton target. This will allow the experiment to measure the Sivers and Boer-Mulders asymmetry in the valence quark region. ${ }^{30}$ There are plans at RHIC to measure prompt photon asymmetry arising due to Sivers mechanism in $p^{\uparrow} p$ collisions. Such an asymmetry is also shown to change sign when compared to the Sivers asymmetry in SIDIS process. ${ }^{31}$ There are other proposals to perform polarized Drell-Yan experiments at GSI in Germany and JINR in Russia.

\section{Summary}

The proposed SIDIS measurements with $12 \mathrm{GeV}$ electron beam at Jefferson Lab will provide the most precise 4-dimensional $\left(x, Q^{2}, z, P_{T}\right)$ mapping of SSA and DSA on both proton and neutron targets. The combined analysis of the data from these measurements will help in extracting various TMDs for both $\mathrm{u}$ and d-quarks. The high precision data in the wide kinematic range will also allow us to study the systematic uncertainties in the extraction of TMDs. The extraction of transversity PDF will also enable us to perform a model independent extraction of the $u$ and d-quark tensor charges. Measurement of Sivers, pretzelosity and $g_{1 T}$ distribution functions will enable a comprehensive understanding of quark orbital angular momentum, its relativistic effects and spin-orbit correlations. The future experiments in hadronic 
collisions $(p-p$ or $\pi-p)$ at different facilities will provide much needed data on Sivers and Boer-Mulders function to check their sign compared to SIDIS process.

\section{Acknowledgments}

This work is supported by U.S. Department of Energy. Jefferson Science Associates, LLC operates the Thomas Jefferson National Accelerator Facility under the contract DE-AC05-06OR23177. This work is also supported in part through funds provided by the U.S. Department of Energy under contract DE-FG02-94ER40818.

\section{References}

1. A. Bacchetta et al., JHEP 02093 (2007).

2. F. Yuan, Phys. Rev. Lett. 100 (2008) 032003.

3. S. Arnold, A. Metz and M. Schlegel, Phys. Rev. D 79 (2009) 034005.

4. D. Sivers, Phys. Rev. D41 (1990) 83.

5. S. J. Brodsky, D. S. Hwang and I. Schmidt, Phys. Lett. B 53099 (2002).

6. J. C. Collins, Phys. Lett. B 53643 (2002).

7. D. Boer, P.J. Mulders and F. Pijlman, Nucl. Phys. B 667201 (2003).

8. A. Airapetian et al., Phys. Rev. Lett. 94 (2005) 012002.

9. A. Airapetian et al., Phys. Lett. B 693 (2010) 11.

10. M. Alekseev et al., Phys. Lett. B 692 (2010) 240.

11. X. Qian et al., Phys. Rev. Lett. 107 (2011) 072003.

12. Y.X. Zhao et al., Phys.Rev. C 90 (2014) 055201.

13. J. Huang et al., Phys. Rev. Lett. 108 (2012) 052001.

14. Y. Zhang et al., Phys. Rev. C 90055209.

15. K. Allada et al., Phys. Rev. C 89(2014) 042201.

16. JLab Experiment E12-09-018, B. Wojtsekhowski et al. (2009).

17. JLab Experiment PR12-11-111, M. Contalbrigo et al. (2011).

18. JLab Experiment PR12-11-109, H. Avakian et al. (2012).

19. JLab Experiment E12-09-002, K. Hafidi et al. (2008).

20. JLab Experiment E12-06-104, R. Ent et al. (2006).

21. JLab Experiment E12-09-017, R. Ent et al. (2008).

22. JLab Experiment E12-10-006, H. Gao et al. (2010).

23. JLab Experiment E12-11-007, J. Huang et al. (2011).

24. JLab Experiment E12-11-108, H. Gao et al. (2012).

25. M. Anselmino and A. Prokudin, private communication.

26. W. Vogelsang and F. Yuan, private communication

27. B. Pasquini, private communication

28. C. Quintans, EPJ Web of Conferences 66, (2014) 06021.

29. Fermilab E-1039, http://inspirehep.net/record/1309534

30. Fermilab E-1027, http://inspirehep.net/record/1216817

31. L. Gamberg and Z-B. Kang, Phys. Lett. B 718 (2012) 181. 